o

doi:10.5004/dwt.2017.0704

\title{
Enhancement of stability and reactivity of nanosized zero-valent iron with polyhydroxybutyrate
}

\section{Stanisław Wacławek ${ }^{\mathrm{a}, *}$, Laura Chronopoulou ${ }^{\mathrm{b}}$, Marco Petrangeli Papini ${ }^{\mathrm{b}}$, Vinod V.T.P. ${ }^{\mathrm{a}}$, Cleofe Palocci ${ }^{b}$, Jaroslav Kupčík ${ }^{c}$, Miroslav Černík ${ }^{a}$}

anstitute for Nanomaterials, Advanced Technologies and Innovation, Technical University of Liberec, Studentská 1402/2, 46117 Liberec 1, Czech Republic, Tel. +420 485353 006; emails: stanislaw.waclawek@tul.cz (S. Wacławek), vinodvellorathekkaepadil@gmail.com (V.V.T.P.), miroslav.cernik@tul.cz (M. Černík)

${ }^{b}$ Department of Chemistry, University of Rome "La Sapienza", Piazzale Aldo Moro 5, 00185 Rome, Italy, emails: laura.chronopoulou@uniroma1.it(L.Chronopoulou),marco.petrangelipapini@uniroma1.it (M. Petrangeli Papini), cleofe.palocci@uniroma1.it (C. Palocci)

'Institute of Inorganic Chemistry of the Czechoslovak Academy of Sciences, v.v.i., Husinec-Řež, 1001, 25068 Rež Czech Republic, email:kupcik@iic.cas.cz (J. Kupčík)

Received 22 June 2016; Accepted 25 August 2016

\section{A B S T R A C T}

Polyhydroxybutyrate (PHB) is a bacterial storage polyester, which is produced directly in the activated sludge process. In the present study, PHB was tested as a scaffold material for stabilization of nanosized zero-valent iron (nZVI). The morphology of the resulting composite was assessed using transmission electron microscopy (TEM) and scanning electron microscopy (SEM) coupled with energy-dispersive X-ray spectroscopy (EDX) analysis. In addition, the surface chemistry and particle stabilities of nZVI and nZVI/PHB were determined with zeta potential analysis and disc centrifuge measurements, respectively. Moreover, the sedimentation rate of bare nZVI was tested and compared with that for nZVI/PHB. Batch experiments further confirmed that nZVI/PHB particles are not only more stable but also more reactive toward perchloroethene (PCE) and $\mathrm{Cr}(\mathrm{VI})$ in comparison with the bare nZVI. These results suggest that PHB can be developed as an inexpensive and environmentally friendly material for the stabilization of nZVI particles.

Keywords: PHB; nZVI; Stabilization; VOC; Remediation; Cr(VI)

${ }^{*}$ Corresponding author.

Presented at the EDS conference on Desalination for the Environment: Clean Water and Energy, Rome, Italy, 22-26 May 2016. 